\title{
Orientia beyond fever and rash (Unusual presentations of scrub typhus)
}

\author{
Avinash Hanbe Rajanna', Swetha Rajoli² \\ ${ }^{1}$ Assistant Professor, Department of Medicine, Bangalore Medical College and Research Institute, KR road, Bangalore, \\ Karnataka, India, ${ }^{2}$ Senior Resident, Department of Anaesthesia, Bangalore Medical College and Research Institute, KR \\ road, Bangalore, Karnataka, India
}

Scrub typhus is a zoonotic disease caused by an obligate intracellular gram-negative bacterium of the family Rickettsiaceae called Orientia tsutsugamushi. We are reporting three cases: Case 1:- A 42 year old male, farmer who presented with fever, rash and pandigital gangrene. Case 2:- A 52 year old female positive for scrub typhus treated came with c/o bilateral lower limb weakness which gradually progressed to upper limb, diagnosed to be AIDP, Case 3:- A 32 year old male presented with fever, maculopapular rash and leukocytoclastic vasculitis in first week of illness proved by biopsy. All three cases were positive for OXK (Weil Felix). Scrub typhus can present with unusual clinical features and complication during the course of illness and cases are reported for its varied presentations.

Key words: AIDP; Orientia tsutsugamushi; Weil Felix

\section{INTRODUCTION}

Scrub typhus is a zoonotic disease caused by an obligate intracellular gram-negative bacterium of the family Rickettsiaceae called Orientia tsutsugamushi. The disease is transmitted to man by the bite of the infected larval form (chigger) of the trombiculid mite. ${ }^{1}$

Scrub typhus is endemic in India, China, Korea, Pakistan, Taiwan, Japan, Thailand, Malaysia, and Northern Australia. ${ }^{2}$

The disease usually presents after an incubation period ranging between 4 and 20 days and the severity of infection can range from mild symptoms and signs to multi-organ failure. The chief target of the organism is the vascular endothelium of various organs. There is inflammation

\section{Access this article online}

Website:

http://nepjol.info/index.php/AJMS DOI: 10.3126/ajms.v12i5.34010

E-ISSN: 2091-0576

P-ISSN: 2467-9100

Copyright (c) 2021 Asian Journal of Medical Sciences

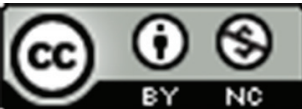

This work is licensed under a Creative Commons Attribution-NonCommercial 4.0 International License. 
Case 1

A 42 year old male, farmer by occupation, presented with history of high grade fever with chills for 7days, which was associated with maculopapular rashes all over body and breathlessness since 1 day.

On physical examination, patient was tachypnoeic with pulse rate of 102 beats/minute and blood pressure 110/80mmHg. He had eschar on the right thigh. Respiratory examination revealed bilateral extensive crepitations. Jugular venous pressure was normal and he was clinically euvolemic. Arterial blood gas revealed type 1 respiratory failure (pH 7.32, pCO2 $45 \mathrm{mmHg}$, PO2 $32 \mathrm{mmol} / \mathrm{l}$ ). In view of progressive breathlessness despite supportive measure, she was intubated and connected to a ventilator. Chest X-ray showed features of acute respiratory distress syndrome (ARDS) (Figure 1). We proceeded with the following investigations to establish the cause for fever with rash and ARDS (Table 1 and 2).

Patient was initially managed with broad spectrum antibiotics - imipenem and linezolid and electrolyte correction. After serology report of scrub typhus being positive (Weil Felix OXK 1:320), he was started on oral doxycycline $100 \mathrm{mg}$ twice daily, following which his fever subsided over 24 hours. On day 3 of admission patient developed blackish discoloration of the terminal digits of upper and lower limbs which were progressive in nature and ultimately developed into dry gangrene (Figures 2) over 4-5 days. However, all his peripheral pulses were felt normally. Vasculitis workup like ANA, Anti dsDNA, antiphospholipid antibody, c ANCA and p ANCA were negative. Surgery opinion was sought and the patient was started on subcutaneous enoxaparin $60 \mathrm{mg}$ twice daily.

After eight days of doxycycline therapy, patient showed gradual improvement in clinical condition

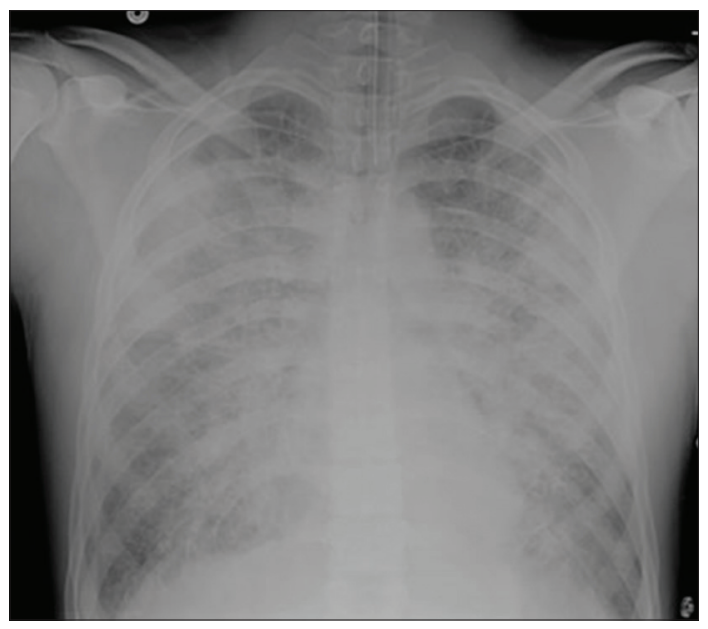

Figure 1: Chest X-ray anterior-posterior view and was weaned off from ventilator subsequently. Patient developed severe pain in upper and lower limb digits around the gangrenous areas. He received several analgesics including tramadol, amitriptyline, paracetamol, NSAIDS with partial relief of pain. He was started on aspirin (150mg OD) and pentoxifylline (400mg TID) by surgeon after cessation of enoxaparin. He eventually developed auto amputation of his left $3^{\text {rd }}$ toe followed by $2^{\text {nd }}$ left toe and little finger of right hand after 2 months.

\section{Case 2}

A 52 year old female, came with complaints of weakness of lower limbs since 5 days and blackish discoloration of left $2^{\text {nd }}$ toe since 4 days. About 10 days prior to the visit, she had consulted at a local hospital for fever and rash. She was diagnosed to have scrub typhus infection (Weil Felix positive for OXK1:320). She was treated with doxycycline. Her symptoms marginally improved, but developed weakness in both lower extremities. She had no other significant past history.

On examination, vital signs were stable (blood pressure $120 / 70 \mathrm{mmHg}$, pulse rate 68 beats/minute, body temperature $\left.36.6^{\circ} \mathrm{C}\right)$. A physical examination revealed

\begin{tabular}{ll} 
Table 1: Laboratory investigations of the patient \\
\hline Haemoglobin & $10 \mathrm{~g} / \mathrm{dl}$ \\
TLC & $14400 \mathrm{cells} / \mathrm{mm}^{3}$ \\
Platelet count & $1,09,000 / \mathrm{mm}^{3}$ \\
Peripheral smear & Normocytic normochromic anaemia \\
& and neutrophilic leucocytosis with shift \\
& to left and thrombocytopenia \\
Serum creatinine & $1.1 \mathrm{mg} / \mathrm{dl}$ \\
Serum sodium & $116 \mathrm{meq} / \mathrm{l}$ \\
Serum potassium & $3.2 \mathrm{mmeq} / \mathrm{l}$ \\
Total bilirubin & $0.7 \mathrm{mg} / \mathrm{dl}$ \\
ALT & $41 \mathrm{U} / \mathrm{L}$ \\
AST & $35 \mathrm{U} / \mathrm{L}$ \\
Alkaline phosphatase & $99 \mathrm{U} / \mathrm{L}$ \\
Serum albumin & $2.2 \mathrm{~g} / \mathrm{dl}$ \\
ECG & Normal \\
\hline
\end{tabular}

\begin{tabular}{ll} 
Table 2: Laboratory investigations \\
\hline $\begin{array}{l}\text { Peripheral smear for malarial } \\
\text { parasite }\end{array}$ & Negative \\
Serology for scrub typhus & Positive (OXK = 1:320) \\
IGM antileptospiral antigen & Negative \\
IGM antibody and Dengue NS1 & Negative \\
HIV & Negative \\
HBsAg & Negative \\
HCV & Negative \\
Blood culture & No growth \\
Urine culture & No growth \\
Echocardiogram & Normal with Ejection fraction \\
& $63 \%$ \\
USG abdomen & Normal \\
\hline
\end{tabular}


lymphadenopathy in the right inguinal area, a maculopapular rash on the chest wall, eschar on the right thigh and gangrene of left second toe, all peripheral pulses felt, patient conscious and oriented power of limbs (upper limb normal; 3/5 in both the lower limb). Lower limb reflexes were diminished with no bladder involvement. Routine laboratory investigations are shown in Table 3.

Lumbar puncture revealed a glucose level of $74 \mathrm{mg} / \mathrm{dl}$ (simultaneous blood glucose- $136 \mathrm{mg} / \mathrm{dl}$ ), a total protein level $210 \mathrm{mg} / \mathrm{dL}$, and white blood cell (WBC) count of 20/mm3 (lymphocytes 90\%). HIV was negative. Workup for gangrene (ANA, c ANCA AND p ANCA) were also negative.

Two days after admission, weakness progressed in the form of upper limb-grade 3/5; lower limb-grade 2/5 and she developed altered consciousness. Nerve conduction study showed diffuse demyelinated neuropathy, which was prominent in the lower extremities. The brain magnetic resonance diffusion image was normal. Neurology opinion sought and oral doxycycline was continued at $100 \mathrm{mg}$ twice daily. On day 4 after admission, the patient complained of dysphagia and dyspnoea. The patient was intubated and

\begin{tabular}{ll} 
Table 3: Laboratory investigation of patient \\
\hline Haemoglobin & $11.8 \mathrm{~g} / \mathrm{dl}$ \\
TLC & $12900 \mathrm{cells} / \mathrm{mm} 3$ \\
Platelet count & $2,98,000 / \mathrm{mm} 3$ \\
Peripheral smear & Normocytic normochromic \\
& blood picture \\
Serum creatinine & $0.9 \mathrm{mg} / \mathrm{dl}$ \\
Serum sodium & $134 \mathrm{meq} / \mathrm{l}$ \\
Serum potassium & $3.7 \mathrm{meq} / \mathrm{l}$ \\
Total bilirubin & $1 \mathrm{mg} / \mathrm{dl}$ \\
ALT(alanine transferase) & $36 \mathrm{U} / \mathrm{L}$ \\
AST(aspartate amino transferase) & $24 \mathrm{U} / \mathrm{L}$ \\
Alkaline phophatase & $147 \mathrm{U} / \mathrm{L}$ \\
Serum albumin & $2.7 \mathrm{~g} / \mathrm{dl}$ \\
ECG & normal \\
\hline
\end{tabular}

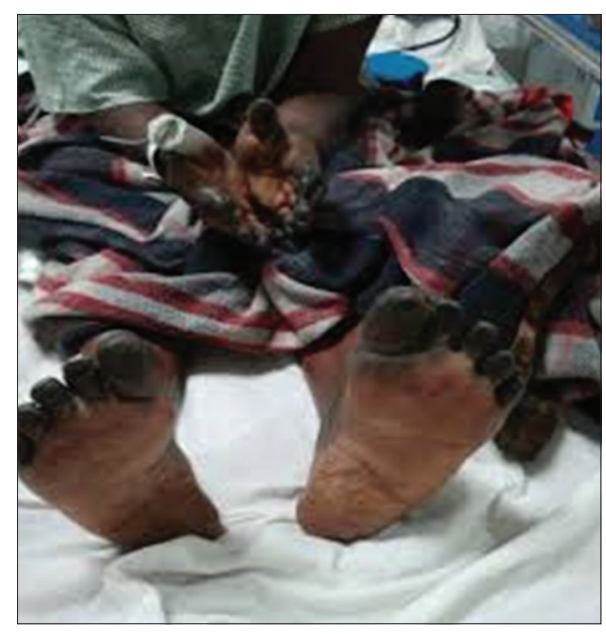

Figure 2: Gangrene involving digits started on intravenous immunoglobin, following which the patient subsequently improved extubated and later discharged.

\section{Case 3}

A 33 year old male came with complaints of, fever with chills of 7 days duration, reddish maculopapular rashes over both the lower limbs and multiple joint pains.

On examination, his pulse rate was 96 beats / minute, blood pressure $130 / 80 \mathrm{mmHg}$ and respiratory rate $20 /$ minute. Skin examination revealed multiple palpable erythematous plaques predominantly distributed over both the lower limbs (Figure 3). The musculoskeletal examination showed calf muscle tenderness and no joint swelling. The other systemic examinations were normal. Laboratory examination revealed normal complete blood count, urine routine, renal and liver function tests.

The Weil Felix (OXK 1:320) was positive. Skin biopsy done on day 2 of hospital admission (day 8 of fever and rash) revealed leukocytoclastic vasculitis (Figure 4). The

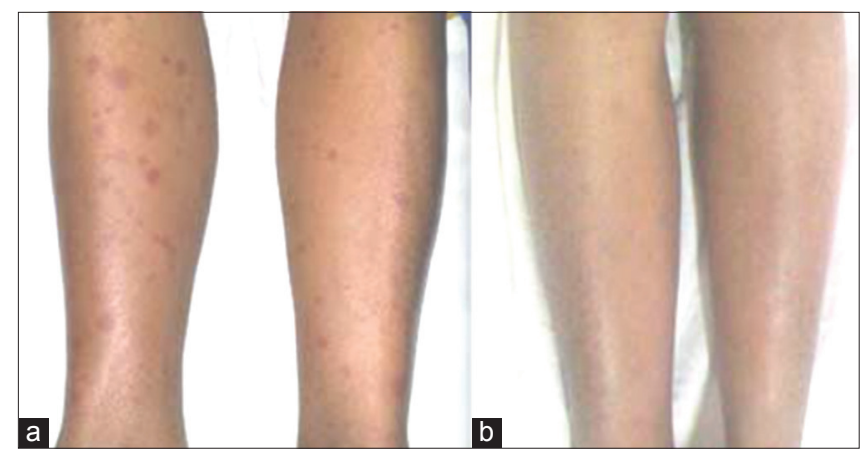

Figure 3: Multiple palpable erythematous plaques distributed over both lower limbs (a) and (b) lesions completely resolved after treatment

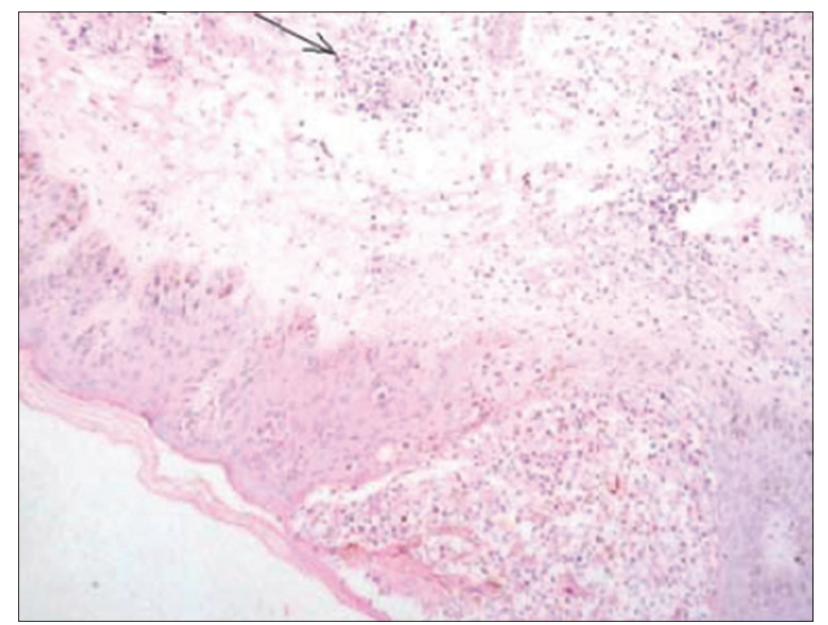

Figure 4: Normal epidermis and the dermis with inflammatory infiltrate, eosinophils and neutrophils. The blood vessels (arrows) infiltrated by inflammatory cells 
blood and urine cultures were sterile. Leptospira serology, Widal test, Dengue serology, HBsAg, HCV and HIV were negative.

\section{DISCUSSION}

Scrub typhus infections may present with fatal complications like ARDS, meningoencephalitis, acute kidney injury, myocarditis, hepatic dysfunction and multiorgan involvement. Complications usually occur after the first week of illness ${ }^{3}$. Due to non- specific clinical features of scrub typhus it is grossly under diagnosed, though it is a growing and emerging disease. Since scrub typhus infection can be life threatening, initiation of treatment should be early and prompt based on clinical suspicion, which later should be confirmed by serology.

O. tsutsugamushi proliferates in the endothelium of small blood vessels that result in cytokine release. This cytokine causes endothelial damage leading to leakage of fluid and aggregation of platelets, and proliferation of polymorph and monocytes leading to focal microinfarction. ${ }^{6,7}$ The Rickettsia attacks the endothelial cells causing inflammation by a mechanism called oxidative stress leading onto local and systemic vasculitis. ${ }^{7}$ Despite the fact that disseminated endothelial dysfunction occurs in scrub typhus infection, the real incidence of vasculitis leading total vaso-occlusion is quite rare which was the highlight of our case.

The importance of differentiating infectious and noninfectious cause of vasculitis is necessary because the treatment strategies are different. While looking into the infective causes as in our case, the diagnosis of scrub typhus should be made by excluding other viral infectious diseases like dengue, infectious mononucleosis, HIV and bacterial diseases like typhoid, leptospirosis, meningococcal disease causing ARDS and vasculitis. These infections were excluded with appropriate investigations. Since the serology for scrub typhus was positive, we conclude that the patient developed pan-digital gangrene secondary to scrub typhus vasculitis.

Guillain-Barré syndrome (GBS) is known to be associated with several infections, such as Campylobacter jejuni, CMV, EBV, and M. pneumoniae. Our cases were diagnosed as scrub typhus based on presence of a maculopapular rash, an eschar, and a positive serology. Additionally, we could diagnose GBS based on neurological examination and nerve conduction study. Molecular mimicry involves the sharing of antigens between the host and an infecting microorganism, and is a type of cross-reactivity similar to an autoimmune disease. Scrub typhus related GBS is suspected to undergo a similar phenomenon. O. tsutsugamushi antibody or antigens presented on infected cells are suspected to activate mimicry on myelin cells or peripheral nerve axons, which elicits immune reactions similar to autoimmune diseases. Scrub typhus associated with GBS rare condition, only three cases of GBS related to scrub typhus have been reported. ${ }^{4,5}$

Our case presented with features of vasculitis and polyarthralgia in the 1 st week of untreated illness, which is very rare and unusual. Hence scrub typhus can present with unusual clinical features during the course of illness and the case series reported for its rare presentation

\section{CONCLUSION}

Vasculitis is one of the dreaded complications of scrub typhus which can lead to digital ischemia and then gangrene, only few cases have been reported, so while dealing with pan digital gangrene, scrub typhus should be kept in mind. Scrub typhus associated with GBS is a rare condition, features of vasculitis and polyarthralgia in the 1st week of illness is quite rare and unusual in scrub typhus.

Scrub typhus can present with unusual clinical features and complications during the course of illness and the cases have been reported for their varied presentations.

\section{ACKNOWLEDGEMENT}

The authors take this opportunity to thank BMCRI for their whole hearted support for this study

\section{REFERENCES}

1. Watt $G$ and Parola P. Scrub typhus and tropical rickettsioses. Current Opinion in Infectious Diseases. 2003; 16(5):429-436. https://doi.org/10.1097/00001432-200310000-00009

2. Hornick RB. Rickettsial Diseases. In: Bennett JC, Plum F, editors. Goldman: Cecil Textbook of Medicine. 21st ed. Philadelphia, USA. WB Saunders Company 2000. pp. 1911-1912.

3. Lee SH, Jung SI, Park KH, Choi SM, Park MH, Kim BC, et al. Guillain-Barré syndrome associated with scrub typhus. Scand J Infect Dis. 2007; 39:826-828.

https://doi.org/10.1080/00365540701266755

4. Lee HS, Lee YJ and Park HY. Guillain-Barre syndrome associatedwith Tsutsugamushi disease. J Korean Neurol Assoc. 2007; 25:275-277.

5. Varghese GM, Abraham OC, Mathai D, Thomas K, Aaron R, 
Kavitha ML, et al. Scrub typhus among hospitalised patients with febrile illness in South India: Magnitude and clinical predictors. J Infect. 2006; 52:56-60.

https://doi.org/10.1016/j.jinf.2005.02.001
6. Cowan G. Rickettsia diseases: The typhus group of fevers--a review. Postgrad Med J. 2000; 76(895):269-272.

7. Mahajan SK. Scrub typhus. J Assoc Physicians India. 2005; 53:954-958.

Author's contribution:

AHR - Concept and design of the study; Interpreted the results; reviewed the literature and manuscript preparation and revision of the manuscript; SR - Statistically analysed and interpreted, preparation of manuscript ; Concept, coordination, review of literature and manuscript preparation.

Work Attributed to:

Bangalore Medical College and Research Institute, KR road, Bangalore, Karnataka, India.

Orcid ID:

Dr. Avinash H R - (D) https://orcid.org/0000-0001-6484-5190

Dr. Swetha Rajoli - (1) https://orcid.org/0000-0002-0765-7959

Source of Funding: None, Conflict of Interest: None. 